\title{
Confirmation of a Gametophytic Self-Incompatibility in Oryza longistaminata
}

\author{
Xiaoping Lian, Shilai Zhang, Guangfu Huang, Liyu Huang, Jing Zhang and Fengyi Hu* \\ State Key Laboratory for Conservation and Utilization of Bio-Resources in Yunnan, Research Center for Perennial Rice \\ Engineering and Technology of Yunnan, School of Agriculture, Yunnan University, Kunming, China
}

\section{OPEN ACCESS}

Edited by:

Kenneth Lee McNally, International Rice Research Institute

(IRRI), Philippines

Reviewed by:

Lijun Chai,

Huazhong Agricultural University,

China

Mulatu Geleta

Swedish University of Agricultural

Sciences, Sweden

Kazuyuki Doi,

Nagoya University, Japan

*Correspondence:

Fengyi Hu

hfengyi@ynu.edu.cn

Specialty section:

This article was submitted to

Plant Breeding,

a section of the journal

Frontiers in Plant Science

Received: 25 June 2020

Accepted: 15 March 2021

Published: 31 March 2021

Citation:

Lian X, Zhang S, Huang G,

Huang L, Zhang J and Hu F (2021)

Confirmation of a Gametophytic

Self-Incompatibility in Oryza

longistaminata.

Front. Plant Sci. 12:576340.

doi: 10.3389/fp/s.2021.576340
Oryza longistaminata, a wild species of African origin, has been reported to exhibit self-incompatibility (SI). However, the genetic pattern of its SI remained unknown. In this study, we conducted self-pollination and reciprocal cross-pollination experiments to verify that $O$. longistaminata is a strictly self-incompatible species. The staining of pollen with aniline blue following self-pollination revealed that although pollen could germinate on the stigma, the pollen tube was unable to enter the style to complete pollination, thereby resulting in gametophytic self-incompatibility (GSI). LpSDUF247, a S-locus male determinant in the gametophytic SI system of perennial ryegrass, is predicted to encode a DUF247 protein. On the basic of chromosome alignment with LpSDUF247, we identified OISS1 and OISS2 as Self-Incompatibility Stamen candidate genes in O. Iongistaminata. Chromosome segment analysis revealed that the Self-Incompatibility Pistil candidate gene of $O$. longistaminata $(O / S P)$ is a polymorphic gene located in a region flanking OISS1. OISS1 was expressed mainly in the stamens, whereas OISS2 was expressed in both the stamens and pistils. OISP was specifically highly expressed in the pistils, as revealed by RT-PCR and qRT-PCR analyses. Collectively, our observations indicate the occurrence of GSI in O. longistaminata and that this process is potentially controlled by OISS1, OISS2, and OISP. These findings provide further insights into the genetic mechanisms underlying self-compatibility in plants.

Keywords: gametophytic, OISP, OISS, Oryza longistaminata, self-incompatibility

\section{INTRODUCTION}

Self-incompatibility (SI) is a reproductive mechanism of higher plants for preventing inbreeding by inhibiting self-pollination, and has been widely observed in Brassicaceae, Solanaceae, Rosaceae, Plantaginaceae, Scrophulariaceae, Gramineae, and Rutaceae (Pandey, 1960; Goring et al., 1993; Vaughan, 1994; Dickinson, 1995; McCormick, 1998; Liu et al., 2014; Iwano et al., 2015; Li et al., 2017; Ye et al., 2018; Liang et al., 2020). SI is described as a mechanism whereby plants are able to produce functional male and female gametes, but are incapable of producing seeds subsequent to self-pollination or the cross-pollination of plants containing SI alleles (Pandey, 1960; Takayama and Isogai, 2005). SI was divided into gametophytic self-incompatibility (GSI) and sporophytic selfincompatibility (SSI), which is defined based on the genetic mode of control of the incompatibility phenotype in pollen (de Nettancourt, 1997; McCubbin and Kao, 2000; Silva and Goring, 2001). In GSI, incompatibility is determined by pollen genotype, where growth inhibition of incompatible 
pollen occurs mainly in the style. In SSI, pollen grain incompatibility is determined by the diploid genome of the parent, in which mature pollen carry SI signal elements secreted by the tapetum, which lead to inhibiting of the germination of incompatible pollen on the stigma surface (de Nettancourt, 1997; Hiscock and Tabah, 2003; Hiscock and McInnis, 2004). The genetic mechanism of SI is dependent on signal transmission controlled by a single or multiple genetic loci between pollen and stigma (Franklin-Tong et al., 1988; Franklin-Tong and Franklin, 2003; Castric and Vekemans, 2004). The genetic loci associated with SI characteristically show high polymorphism, with respect to both the number of $S$ alleles and differences in nucleotide sequences (Vekemans and Slatkin, 1994).

The SI system in Gramineae is GSI, which is thought to be controlled by two non-linked and polymorphic multiallelic $S$ and $Z$ loci (Lundqvist, 1954; Cornish et al., 1979; Baumann et al., 2000; Thorogood et al., 2002). The $S$ and $Z$ loci in Lolium perenne L. have been found to be correspond to syntenic regions of chromosomes 5 and 4 in the genome of O. sativa, respectively (Yang et al., 2009). In total, 10 SI candidate genes have been identified in the SI cDNA libraries of L. perenne, and qRT-PCR analysis has revealed a rapid increase in the expression of these genes following pollenstigma contact (Yang et al., 2009). Subsequently, nine candidate genes associated with SI were screened from $L$. perenne using molecular markers, and on the basis of expression profiling and nucleotide diversity assessment, two $Z$-linked genes, a ubiquitinspecific protease and a LPDUF247 gene, have been identified as the most plausible candidates for the $S-Z$ SI system (Shinozuka et al., 2010). In further studies, a total of 10,177 individuals of L. perenne from seven different mapping populations segregating for $S$ locus showed a highly polymorphic gene encoding for a protein containing a DUF247 was fully predictive of known $S$-locus genotypes at the amino acid level (Manzanares et al., 2016). In addition, genome wide association studies and BLAST alignment with the Brachypodium physical map revealed highly significantly associated markers with peak associations from the chromosomal locations of candidate SI genes $S$ - and Z-DUF247 (Thorogood et al., 2017). LpSDUF247 is considered to be a $S$-locus male determinant in the gametophytic SI system that has five haplotypes with sequence polymorphism, which is a typical characteristic of SI. However, the molecular mechanisms underlying the regulation of SI in L. perenne have yet to be clarified (Thorogood et al., 2002; Shinozuka et al., 2010; Manzanares et al., 2016).

Oryza longistaminata is an AA-genome wild Oryza species of African origin that exhibits SI (Nayar, 1967). O. longistaminata has numerous desirable traits, such as long stigma and large anthers (Morishima, 1967), a rhizome (Ghesquiere, 2008), and high resistance to abiotic and biotic stresses (Khush et al., 1990; Song et al., 1995; Hu et al., 2003; Dong et al., 2017), and accordingly could represent a beneficial resource from the perspective of rice breeding. However, SI limits its free mating and hinders the exploitation of such desirable traits in rice cultivar improvement. In the present study, we identified a polymorphic $S$-locus associated with SI in this species, which will provide a basis for the determination of genetic patterns and molecular mechanisms of SI. Accordingly, we believe that the findings of this study could potentially facilitate exploitation of the desirable traits of O. longistaminata.

\section{MATERIALS AND METHODS}

\section{Plant Materials}

For the determining the SI of O. longistaminata, we used the germplasms OlMK23 and OlMK68, which were originally collected from Ethiopia and were kindly provided by Dr. Melaku Getachew of the Institute of Biotechnology, College of Natural and Computational Sciences, Addis Ababa University, Ethiopia. For reciprocal hybrid testing, OlMK68 and OlMK23 plants were cultivated at Jinghong, Yunnan Province, China $\left(21^{\circ} 59^{\prime} \mathrm{N}, 87100^{\circ} 44^{\prime} \mathrm{E}, 611 \mathrm{~m}\right.$ a.s.l.). For hybridization experiments, stamens were removed manually from the pollen recipients and mature pollen was collected from the pollen donors.

\section{Reciprocal Hybrids and Statistics}

The O. longistaminata germplasms OlMK23 and OlMK68, and their reciprocal hybrids, each of which was represented by 10 samples, were cultivated for SI testing. For the reciprocal hybrids, the stamens were removed from a pollen recipient and mature pollen grains were collected from a pollen donor, after which plant were cross-pollinated and bagged. The seed-setting rate of OlMK23, OlMK68, and their reciprocal hybrids was determined after harvesting. Statistical analyses of seed-setting rate were based on the ratio between the number of harvested mature seeds and the total number of pollinated flowers in 10 independent pollination experiments.

\section{Aniline Blue Staining}

At $5 \mathrm{~min}, 30 \mathrm{~min}, 1 \mathrm{~h}$, and $2 \mathrm{~h}$ following self-/cross-pollination, more than 10 pollinated $O$. longistaminata and $O$. sativa pistils were collected and subsequently fixed overnight in Carnoy's solution ( $90 \mathrm{~mL}$ of $50 \%$ ethanol, $5 \mathrm{~mL}$ of formaldehyde, and $5 \mathrm{~mL}$ of acetic acid). The pistils were then sequentially rehydrated with ethanol (50,30, and $10 \%$, each for $10 \mathrm{~min}$ ) and then washed with deionized water. Thereafter, the pistils were softened in $1 \mathrm{M} \mathrm{NaOH}$ for $45 \mathrm{~min}$ at $55^{\circ} \mathrm{C}$ and stained with water-soluble aniline blue $[0.1 \%(\mathrm{~W} / \mathrm{V})$ aniline blue and $0.1 \mathrm{M} \mathrm{K} \mathrm{K}_{2} \mathrm{HPO}_{4}, \mathrm{pH}$ 8.5] for $6 \mathrm{~h}$ in the dark at room temperature. The stained pistils were visualized by fluorescence microscopy.

\section{Identified and Syntenic Analysis of OISSs and OISP}

LpSDUF247 is a male component of the S-locus in L. perenne and is syntenic with O. sativa (Manzanares et al., 2016). OsDUF247 (Os05g0197900), which corresponds to LpSDUF247, is located in the 6,030-6,090 $\mathrm{kb}$ region of $O$. sativa chromosome A05. Comparative genomics analyses were performed by comparing 
the genome sequences of O. longistaminata with chromosome 5 of $O$. sativa. OlSS1 on Contig10 of O. longistaminata corresponds to OsDUF247 (Os05g0197900). Sequence analysis was performed on the sequence flanking OlSS1 to identify candidate polymorphic sequences. Haplotype and sequence polymorphism analyses of OlSS1, OlSS2, and OlSP were based on published transcriptome sequencing data (Zhang et al., 2015), whereas analysis of the conserved domain of OlSS1, OlSS2, and OlSP was performed using SMART ${ }^{1}$.

\section{RNA Isolation}

The roots, stems, leaves, stamens, and pistils of $O$. longistaminata were used for tissue-specific expression analysis. The stamens were collected to determine the expression of OlSS1 and OlSS2 from stages 7 to 14 and expression in the pistils was determined from stages 9 to 14 as described by Zhang and Zoe (2009) for O. sativa. Total RNA was isolated from O. longistaminata using an Eastep ${ }^{\circledR}$ Super Total RNA Extraction Kit (Promega, Shanghai, China), with first-strand cDNA being synthesized from $1 \mu \mathrm{g}$ of the isolated RNA using a PrimeScript RT Reagent Kit with gDNA Eraser (TaKaRa BIO, Shiga, Japan).

\section{RT-PCR and qRT-PCR Analysis}

The cDNAs of roots, stems, leaves, stamens, and pistils of O. longistaminata were used as templates for RT-PCR assays. RT-PCR and qRT-PCR amplifications were performed using the following primer pairs: OlSS1-1-F and OlSS1-1-R for OlSS1-1; OlSS1-2-F and OlSS1-2-R for OlSS1-2; OlSS2-1-F and OlSS2-1$\mathrm{R}$ for OlSS2-1; OlSS2-2-F and OlSS2-2-R for OlSS2-2; OlSP-1-F and OlSP-1-R for OlSP-1; and OlSP-2-F and OlSP-2-R for OlSP2. qRT-PCR analysis was performed using SYBR Premix Ex Taq Kit (TaKaRa BIO) in a Quant Studio 7 Flex real-time PCR system (Applied Biosystems, Foster City, CA, United States) with the following amplification program: initial denaturation at $95^{\circ} \mathrm{C}$ for $30 \mathrm{~s}$, followed by 40 cycles of denaturation at $95^{\circ} \mathrm{C}$ for $5 \mathrm{~s}$ and primer annealing and extension at $58^{\circ} \mathrm{C}$ for $34 \mathrm{~s}$. The results were normalized using the relative expression level of Actin 2 and analyzed using the $2^{-\Delta} \Delta C t$ method (Livak and Schmittgen, 2001). Three biological replicates were performed for RT-PCR and qRT-PCR analysis. The sequences of the primers used in this study are presented in Supplementary Table 1.

\section{Homologous Cloning of OISSs and OISP}

Relevant genomic and transcriptomic data for homologous cloning obtained from a previous study on O. longistaminata (Zhang et al., 2015). The cDNA sequences of OlSSs and OlSP were cloned from the transcriptomic database of O. longistaminata by homologous cloning (Zhang et al., 2015). For cDNA cloning, we used the following primers pairs: OlSS1-1-cF and OlSS1-1-cR were used for OlSS1-1; OlSS1-2-cF and OlSS1-2-cR for OlSS1-2; OlSS2-1-cF and OlSS2-1-cR for OlSS2-1; OlSS2-2-cF and OlSS2-2$c R$ for OlSS2-2; OlSP-1-cF and OlSP-1-cR for OlSP-1; and OlSP-2$c F$ and OlSP-2-cR for OlSP-2. The products thus amplified were inserted in the $p M D 19-T$ vector, and monoclonal sequencing was performed for different haplotypes.

${ }^{1}$ http://smart.embl.de/

\section{RESULTS}

\section{Oryza longistaminata Is a Strictly Self-Incompatible Species}

The definitive characteristic of SI is that self-pollination of identical haplotypes results in sterility, whereas pollen is fertile when crossing different haplotypes (Franklin-Tong et al., 1988; Franklin-Tong and Franklin, 2003). In the present study, we grew plants of OlMK68 and OlMK23 in a field to evaluate the SI in O. longistaminata through self-pollination and reciprocal cross-pollination. Although the seed-setting rate of the hybrid (OlMK68/OlMK23) and its reciprocal hybrid was $20.5 \pm 3.8$ and $26.2 \pm 4.7 \%$, respectively $(n=10)$, in the case of both OlMK23 and OlMK68, self-pollination of plants failed to result in seed set, suggesting strict SI in this species (Table $\mathbf{1}$ and Supplementary Table 2).

\section{Oryza longistaminata Exhibits Gametophytic Self-Incompatibility}

To determine pollen phenotype, we performed staining with aniline blue, which revealed that the pollen of $O$. longistaminata is able to germinate on the stigma after self-pollination (Figures 1A-D), with germination being observed within $5 \mathrm{~min}$ after self-pollination of OlMK68 (Figure 1A), a high rate of germination after $30 \mathrm{~min}$, and the pollen tube reaching the top of the style within $1 \mathrm{~h}$ (Figures 1B,C). Subsequently, however, pollen tube growth was inhibited, with the pollen tube appearing to be incapable of passing through the style (Figure 1D). In contrast, pollen growth was consistent in selfpollinated of $O$. sativa after 5 and $30 \mathrm{~min}$ (Figures 1E,F), and the pollen tube was observed to have passed through the style within 1-2 h (Figures 1G,H). These results indicated that although the pollen of $O$. longistaminata is able to germinate on the stigma after self-pollination, it is incapable of subsequent passage through the style, there implying that this species exhibits GSI.

\section{Oryza longistaminata Self-Incompatibility Determinants Are Linked S-Loci}

The corresponding region of the $S$-locus of $L$. perenne was found on chromosome 5 of $O$. sativa, wherein a gene homolog of LPSDUF247 is predicted to encode a DUF247 domaincontaining protein (Manzanares et al., 2016). Comparative genomic analysis revealed that a gene located on contig10 of O. longistaminata is syntenic with Os05g0197900 (OsDUF247),

TABLE 1 | Seed-setting rate after self-/cross-pollination in O. Iongistaminata.

\begin{tabular}{ccc}
\hline Female & Male & Seed-setting rate (\%) \\
\hline OIMK68 & OIMK68 & 0 \\
OIMK68 & OIMK23 & $20.5 \pm 3.8$ \\
OIMK23 & OIMK23 & 0 \\
OIMK23 & OIMK68 & $26.2 \pm 4.7$ \\
\hline
\end{tabular}




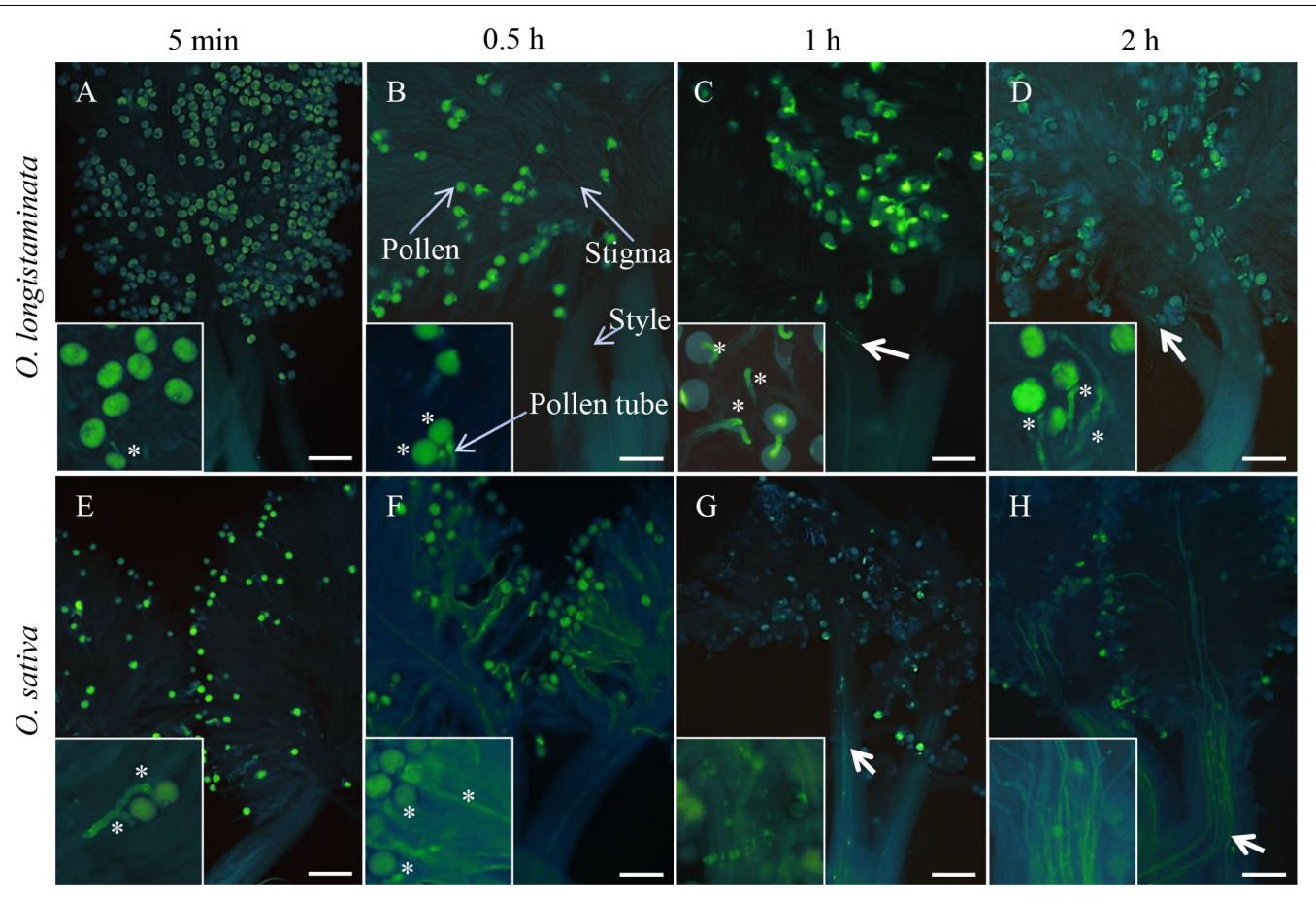

FIGURE 1 | Pollen germination of Oryza longistaminata and Oryza sativa. (A-D) Self-pollination of O. longistaminata: (A) The pistils of self-pollinated OIMK68 after $5 \mathrm{~min}$, stained with aniline blue solution. (B) The majority of pollen grains had germinated at $0.5 \mathrm{~h}$ following self-pollination. (C) The pollen tube reached the top of the style at $1 \mathrm{~h}$ after self-pollination. (D) At $2 \mathrm{~h}$ after pollination, the pollen tube remained on top of the style and appeared incapable of passing through the style. (E-H) Self-pollination of $O$. sativa: (E) Pollen began to germinate 5 min after self-pollination. (F) The pollen tube began to elongate at $0.5 \mathrm{~h}$ after self-pollination and passed through the style within 1-2 $\mathrm{h}$ following cross-pollination $\mathbf{( G , H ) . ~ B a r ~}=50 \mu \mathrm{m}$. The asterisk indicates the germination of pollen grains. Bold arrows indicate pollen tubes in the style.

named Self-Incompatibility Stamen1 of O. longistaminata (OlSS1) (Figure 2A). OlSS1 encode a DUF247 domain protein, which is similar to LpSDUF247 and considered to be involved in SI (Figure 2B). On the basis of homologous sequence alignment, a gene was predicted to encode a protein containing a DUF247 domain about $19 \mathrm{~kb}$ downstream of OlSS1, named Self-Incompatibility Stamen 2 of O. longistaminata (OlSS2) (Figures 2A,B). Previous genomic and transcriptomic sequencing has revealed that both OlSS1 and OlSS2 have two alleles in O. longistaminata (Zhang et al., 2015). RTPCR assays indicated that alleles OlSS1-1 and OlSS1-2 are expressed primarily in the stamens, whereas the OlSS21 and OlSS2-2 alleles are expressed in both stamens and pistils (Figure 2C and Supplementary Figure 1). A defining characteristic of SI is that there should be a close linkage between male and female determinants and expression in stamens and pistils (Franklin-Tong, 2008). Both OlSS1 and OlSS2 show sequence polymorphisms and are expressed in stamens, we speculated that there is a Self-Incompatibility Pistil factor on contig10 of O. longistaminata. Indeed, subsequent sequence polymorphism analysis of a region flanking OlSSs revealed a candidate gene for such a factor, named Self-Incompatibility Pistil factor (OlSP), which may be involved in the SI of O. longistaminata (Figure 2A), given OlSP has a pair of alleles, OlSP-1 and OlSP-2, that are highly and specifically expressed in the pistil (Figure 2C and Supplementary Figure 1). Structural analysis revealed that OlSP encodes an N-terminal YfaZ domain of unknown function (Figure 2B). Consequently, given that OlSSs and OlSP are closely linked and highly expressed in the stamens or pistils of $O$. longistaminata, we believe that OlSSs and OlSP could be candidate genes controlling SI in this species.

The alignment of OlSS1-1 and OlSS1-2 amino acid sequences with that of OsDUF247 exhibited similarity level of 80.7 and $69.8 \%$, respectively (Figure 3), whereas the sequences of OlSS2-1 and OlSS2-2 showed lower similarities of 38.9 and $36.9 \%$, respectively (Figure 3). Both OlSS1 and OlSS2 are characterized by conserved regions at the $\mathrm{N}$ and $\mathrm{C}$ termini. Furthermore, we established that similarities between the amino acid sequences of OlSP1 and OlSP2 and Os05g0198000 are 51.8 and $63.2 \%$, respectively (Figure 4). These findings thus indicate that the OlSS1, OlSS2, and OlSP exhibit sequence polymorphism and can be considered putative SI candidate genes in O. longistaminata.

\section{OISSs and OISP Expression Patterns}

The expression levels of OlSS1, OlSS2, and OlSP at different development stages of stamen and pistil were analyzed by qRTPCR assay. The expression of OlSS1-1 was down-regulated during all developmental stages of the stamens, whereas the expression of OlSS1-2 was up-regulated in stages 7-9 and 
A

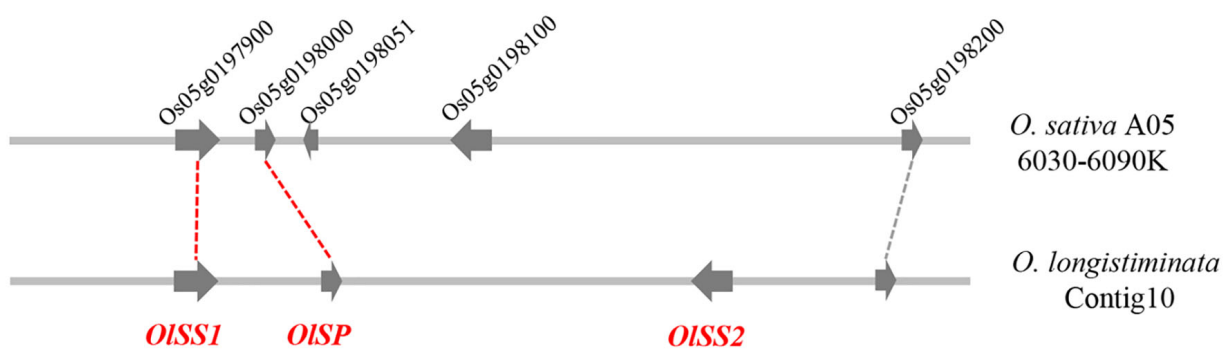

B

\section{C}

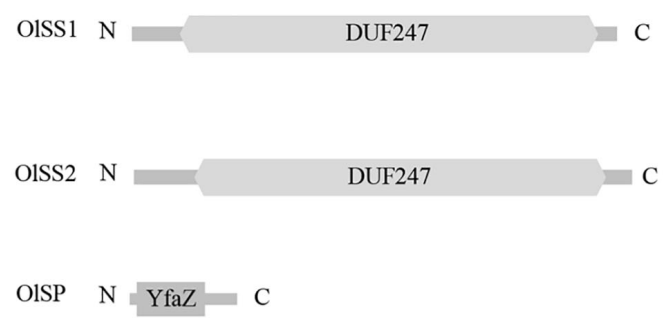

$100 \mathrm{AA}$

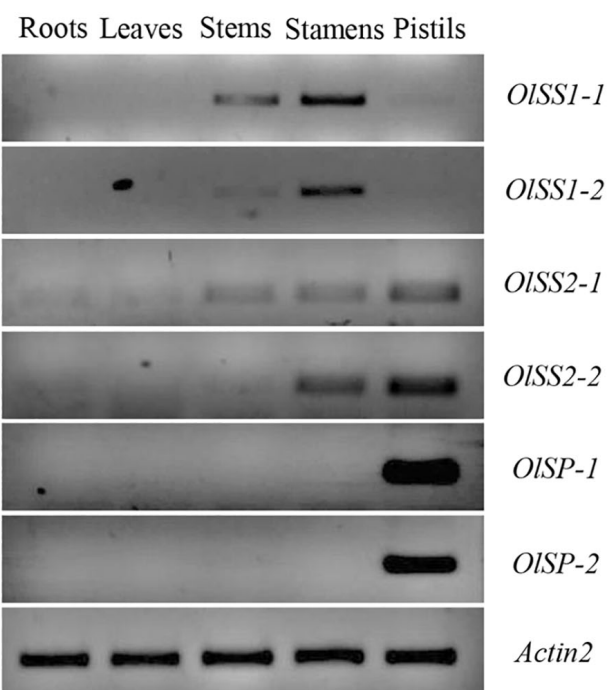

FIGURE 2 | Syntenic and tissue expression of OISSs and OISP. (A) Syntenic representation of OISSs and OISP in Oryza sativa and Oryza longistaminata. Os05g0197900, which encodes an unknown DUF247 domain protein in O. sativa, corresponds to OISS1, whereas Os05g0198000 and Os05g0198100 correspond to OISP and OISS2 respectively. (B) Analysis of OISS1, OISS2, and OISP protein domains. Both OISS1 and OISS2 contain a conserved DUF247 domain, whereas OISP contains an N-terminal YfaZ domain of unknown function. (C) RT-PCR determination of the tissue-specific expression pattern of OISSs and OISP in O. Iongistaminata. OISS1-1 and OISS1-2 are mainly expressed in stamens, whereas OISS2-1 and OISS2-2 are expressed in both stamens and pistils, and OISP-1 and OISP-2 are highly and specifically expressed in pistils. OISS1: Self-incompatibility stamen1 from O. Iongistaminata; OISS2: Self-incompatibility stamen2 from O. Iongistaminata; OISP: Self-Incompatibility Pistil factor from O. Iongistaminata; O. sativa A05: Oryza sativa chromosome 5.

thereafter gradually down-regulated in stages 9-14 (Figure 5A). Interestingly, the expression OlSS2 in stamens was found to show a pattern similar to that of OlSS1, with the downregulated expression of OlSS2-1 started commencing from stage 10 (Figure 5B). Of the two OlSS2 alleles, whereas OlSS2-2 was up-regulated during pistil development but the expression of OlSS2-1 no significant change (Figure 5C). Moreover, both $O l S P-1$ and OlSP-2 were rapidly up-regulated during pistil development, with highest expression levels being detected at stage 13 (Figure 5D).

Analysis of the expression profiles of OlSP-1, OlSP-2, OlSS2-1, and OlSS2-2 after self-/cross-pollination revealed that the expression of OlSP-1 was highest $5 \mathrm{~min}$ after pollination and increased gradually within $1 \mathrm{~h}$ after crosspollination (Figure 5E). Contrastingly, OlSP-2 exhibited no significant change following self-pollination, although showed an expression pattern similar to that of OlSP-1 following crosspollination (Figure 5F). Expression of OlSS2-1 was found to be highest at $30 \mathrm{~min}$ after self-pollination, whereas no significant change following cross-pollination (Figure 5G). OlSS2-2 was up-regulated within $30 \mathrm{~min}$ and subsequently down-regulated after self-pollination, whereas following crosspollination, highest expression levels were detected within 5 min, after which expression was down-regulated (Figure $\mathbf{5 H}$ ). These observations indicate that whereas OlSS1 and OlSP are expressed in the stamens and pistils, respectively, OlSS2 is expressed in both stamens and pistils, thereby indicating that these three genes are associated with stamen and pistil development. In addition, the results revealed that OlSS1, OlSS2, and OlSP have different expression patterns after self-/crosspollination.

\section{DISCUSSION}

Studies on the genetics of SI in Gramineae were mainly conducted on Secale cereale and Phalaris coerulescens, and have indicated that SI in grass species is under 


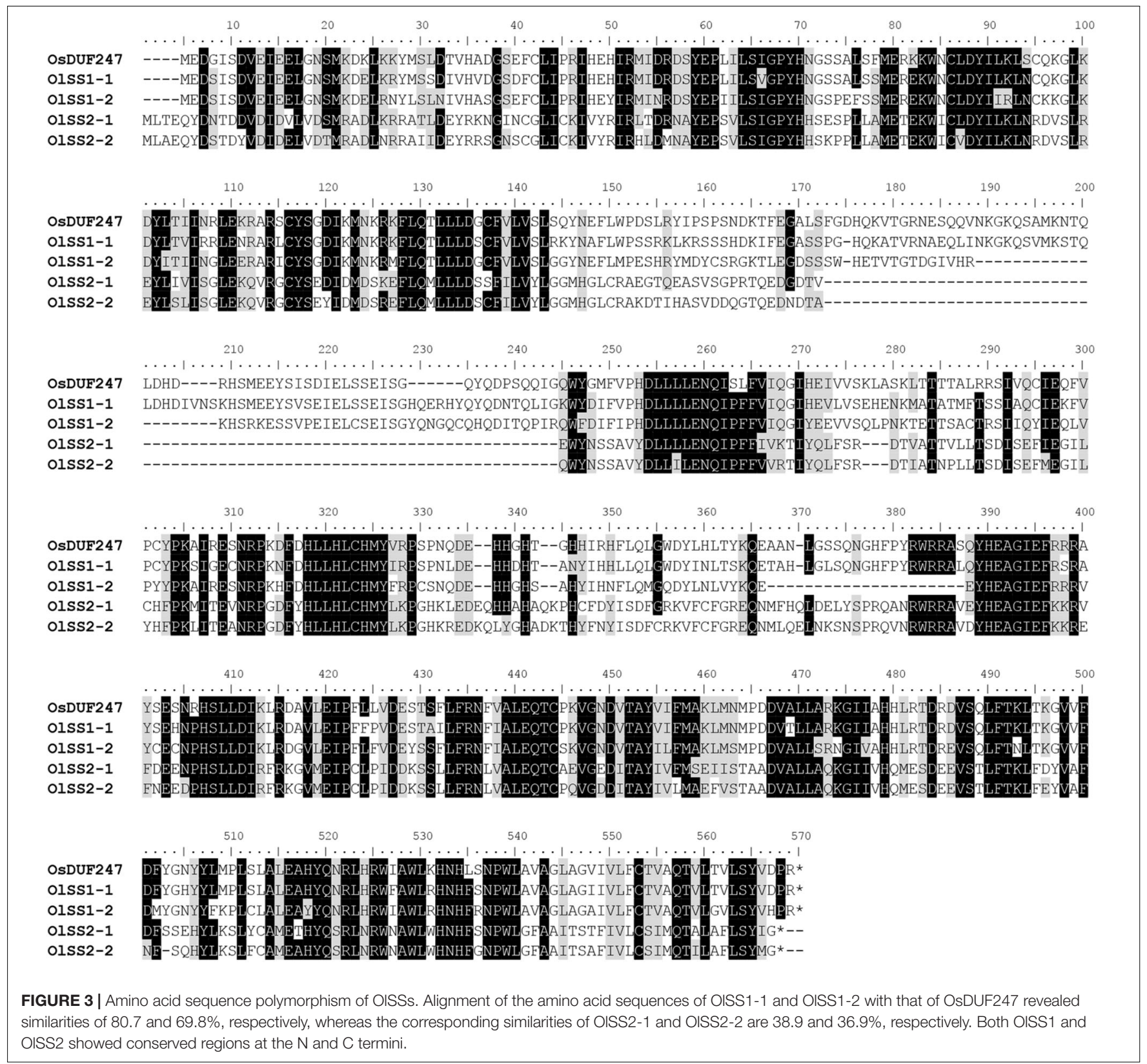

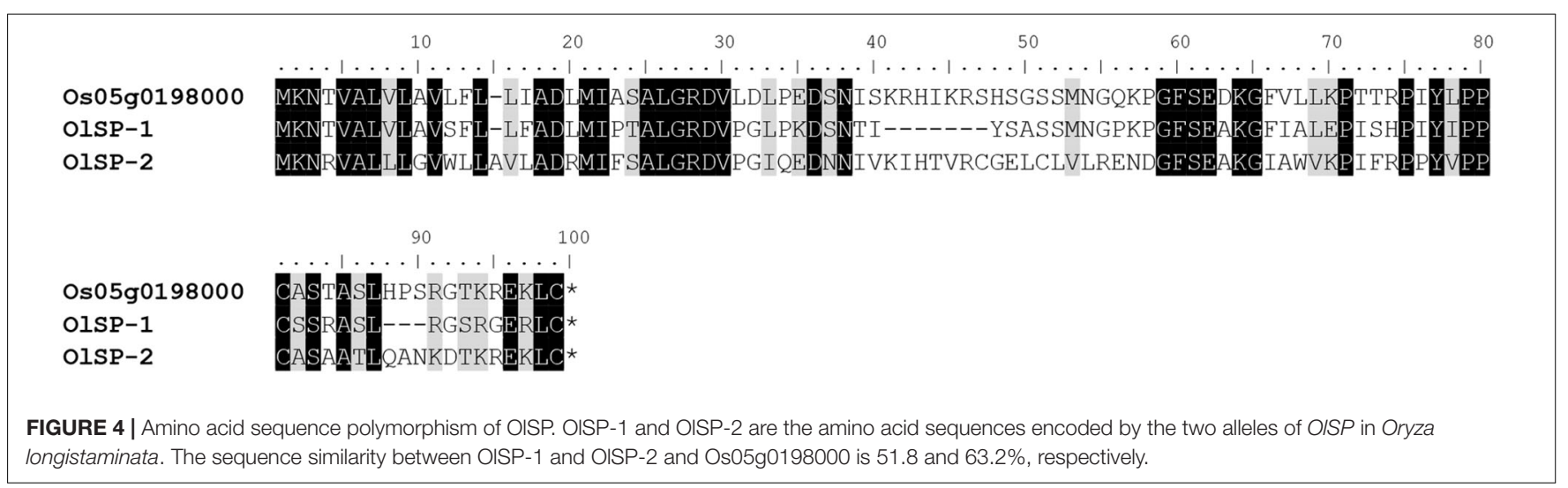


A

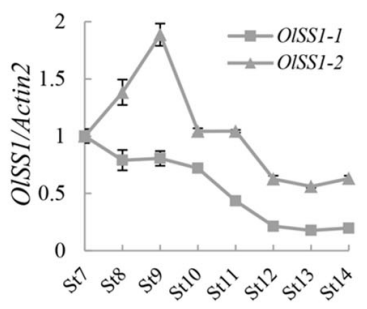

E

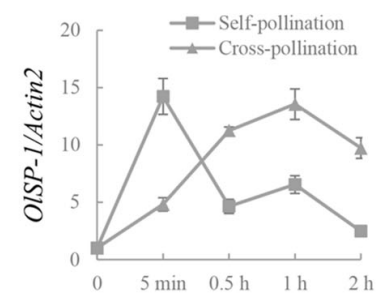

B

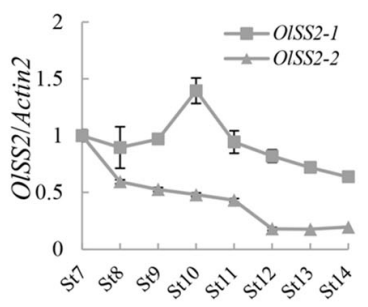

F

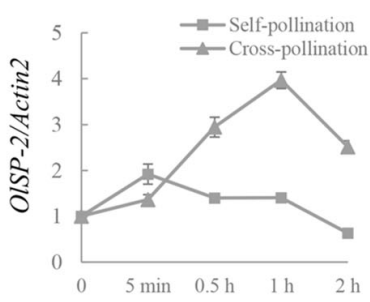

C

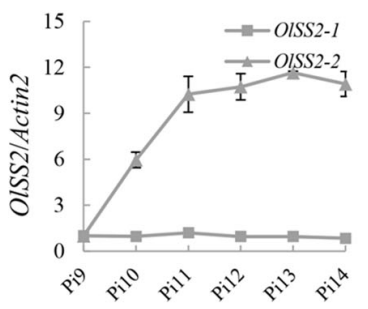

G

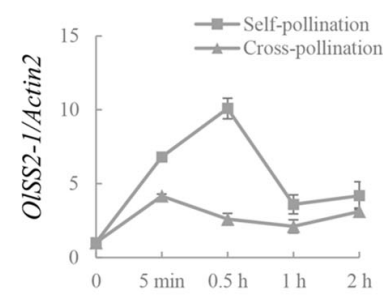

D

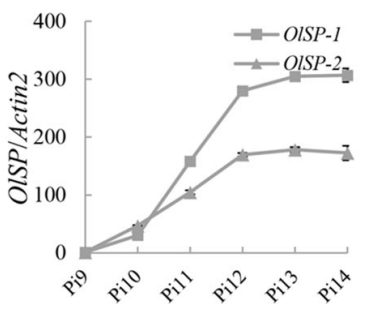

H

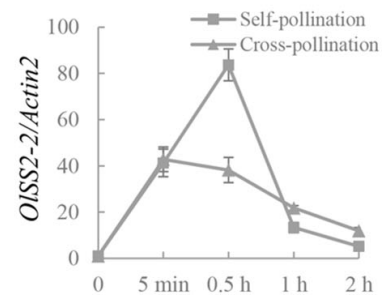

FIGURE 5 | The expression pattern of OISSs and OISP. (A) Expression profile of OISS1 from stage 7 to stage 14 in stamens. OISS1-1 was down-regulated at all stamen developmental stages, whereas OISS1-2 was up-regulated at stages 7-9, and gradually down-regulated from stages 9 to 14. (B) The expression pattern of OISS2 from stage 7 to 14 in stamens. The down-related expression of OISS2-1 commenced at stage 10. (C) The expression pattern of OISS2 in pistils. OISS2-2 up-regulated during pistil development, whereas we detected no significant changes in the expression of OISS2-1. (D) The expression profile of OISP in pistils. OISP-1 and OISP-2 were up-regulated in pistils. (E) The expression profile of O/SP-1 in pistils after self-/cross-pollination. The down-regulated expression of O/SP-1 commenced $5 \mathrm{~min}$ after self-pollination, and reached the highest level at $1 \mathrm{~h}$ after cross-pollination. (F) The expression profile of OISP-2 in pistils after self-/cross-pollination. OISP-2 showed significant changes in expression following self-pollination, but was up-regulated after cross-pollination, attaining the highest level after $1 \mathrm{~h}$. (G) The expression profile of OISS2-1 in pistils after self-/cross-pollination. OISS2-1 was up-regulated within $0.5 \mathrm{~h}$ of self-pollination, after which the levels of expression began to decline. In contrast, no significant changes were detected in the expression of OISS2-1 following cross-pollination. (H) The expression profile of O/SS2-2 in pistils after self-/cross-pollination. O/SS2-2 was up-regulated within $0.5 \mathrm{~h}$ of self-pollination, and subsequently down-regulated. Following cross-pollination, the highest level of OISS2-2 expression was detected within 5 min, after which expression was down-regulated. St, stamen; Pi, pistil; St7 to St14, pollen development from stages 7 to 14; Pi9 to Pi14, pistil development from stages 9 to 14 .

gametophytic control mediated by two unlinked multiallelic loci referred to as $S$ and $Z$ (Lundqvist, 1954, 1956). Available data indicate that the two-locus SI system is common to all self-incompatible grass species of the subfamily Pooideae, and possibly to all members of the family Gramineae (Connor, 1979). The most significant differences with regards to SI are reciprocal differences in compatibility between two parents (Leach, 1988). In the present study, examination of the compatibility between the two investigated $O$. longistaminata germplasms (OlMK68 and OlMK23) revealed complete infertility following selfpollination and different seed-setting rates in reciprocal hybrids, indicating strict SI.

In SI, the transmission of signals between the stamens and pistils is dependent on the interaction of $S$-haplotypes with polymorphic sequences (Franklin-Tong et al., 1988; Franklin-Tong and Franklin, 2003). Therefore, putative specificity determinants should meet the following three criteria suggested by the biology and genetics of SI: linkage to the $S$-locus, polymorphism between different $S$-haplotypes, and expression in the pollen or pistil (Franklin-Tong, 2008). In the present study, the sequence polymorphisms and expression patterns of OlSSs and OlSP were in accordance with the characteristics of SI. Moreover, OlSSs and OlSP were found to be closely linked on the
O. longistaminata genome, which is consistent with the signaling mechanism of SI.

The primer pairs used in this study were designed based on the allele sequences of OlSSs and OlSP from OlMK68, and on the basis of the tissue-specific expression and expression levels in stamens and pistils at different stages of developmental, we confirmed that OlSSs and OlSP are polymorphic loci. Moreover, qRT-PCR analysis revealed that the alleles of OlSS1 and OlSS2 show identical expression patterns in stamens, thereby indicating the comparable or synergistic functions of OlSS1 and OlSS2. Given that the expression of OlSS1 was gradually downregulated during the latter stages of pollen maturation, further examination of the expression of this gene at the cellular level will be necessary to more precisely determine whether OlSS1 is expressed in the pollen or in the tapetum. Besides, OlSS2 was also expressed in the pistil, but only the OlSS2-2 allele is up-regulated during pistil development. The reason for this phenomenon could be indictive of the complex mechanism of SI in O. longistaminata, which requires further study. A notable observation in the present study was that pollen on the stigma was unable to enter the style following self-pollination, indicating that either the pollen carries a male SI signal factor and/or that a pistil signal factor is secreted into the style. Although 
OlSSs and OlSP are considered to be the respective candidate genes for these factors in the SI of O. longistaminata, the mechanisms whereby they contribute to the regulation SI need to be further studied.

\section{DATA AVAILABILITY STATEMENT}

The raw data supporting the conclusions of this article will be made available by the authors, without undue reservation.

\section{AUTHOR CONTRIBUTIONS}

$\mathrm{FH}$ and XL designed the experiments and wrote the manuscript. $\mathrm{XL}, \mathrm{SZ}$, and JZ performed the experiments. GH and LH analyzed the data. All authors have read and agreed to the submitted version of the manuscript.

\section{REFERENCES}

Baumann, U., Juttner, J., Bian, X., and Langridge, P. (2000). Self-incompatibility in the grasses. Ann. Bot. 85, 203-209. doi: 10.1006/anbo.1999.1056

Castric, V., and Vekemans, X. (2004). Plant self-incompatibility in natural populations: a critical assessment of recent theoretical and empirical advances: self-incompatibility in natural plant populations. Mol. Ecol. 13, 2873-2889. doi: 10.1111/j.1365-294X.2004.02267.x

Connor, H. E. (1979). Breeding systems in the grasses: a survey. N. Z. J. Bot. 17, 547-574. doi: 10.1080/0028825X.1979.10432571

Cornish, M. A., Hayward, M. D., and Lawrence, M. J. (1979). Self-incompatibility in diploid Lolium perenne L. Heredity 43, 95-106. doi: 10.1038/HDY.1976.107

de Nettancourt, D. (1997). Incompatibility in Angiosperms. Berlin: Springer. doi: 10.1007/978-3-662-12051-4

Dickinson, H. (1995). Dry stigmas, water and self-incompatibility in Brassica. Sex. Plant Reprod. 8, 1-10. doi: 10.1007/bf00228756

Dong, L., Liu, S., Xu, P., Deng, W., and Yang, Q. (2017). Fine mapping of Pi57(t) conferring broad spectrum resistance against magnaporthe Oryzae in introgression line IL-E1454 derived from Oryza longistaminata. PLoS One 12:e0186201. doi: 10.1371/journal.pone.0186201

Franklin-Tong, N., and Franklin, F. C. H. (2003). Gametophytic selfincompatibility inhibits pollen tube growth using different mechanisms. Trends Plant Sci. 8, 598-605. doi: 10.1016/j.tplants.2003.10.008

Franklin-Tong, V. (2008). Self-incompatibility in Flowering Plants. Berlin: Springer. doi: 10.1007/978-3-540-68486-2

Franklin-Tong, V. E., Franklin, M. J., and Franklin, C. H. (1988). An in vitro bioassay for the stigmatic product of the self-incompatibility gene in papaver rhoeas L. New Phytol. 110, 109-118. doi: 10.2307/2434796

Ghesquiere, A. (2008). "Evolution of Oryza longistaminata," in Rice Genetics I: (In 2 Parts), ed. S. J. Banta (Manila: International Rice Research Institute), 15-25. doi: 10.1142/9789812814265_0002

Goring, D. R., Glavin, T. L., Schafer, U., and Rothstein, S. J. (1993). An S receptor kinase gene in self-compatible Brassica napus has a 1-bp deletion. Plant Cell 5, 531-539. doi: 10.2307/3869708

Hiscock, S. J., and McInnis, S. M. (2004). Pollen recognition and rejection during the sporophyticself-incompatibility response: Brassica and beyond. Trends Plant Sci. 12, 606-613. doi: 10.1016/j.tplants.2003.10.007

Hiscock, S. J., and Tabah, D. A. (2003). The different mechanisms of sporophytic self-incompatibility. Philos. Trans. R. Soc. B 358, 1037-1045. doi: 10.1098/rstb. 2003.1297

Hu, F., Tao, D., Sacks, E., Fu, B., Xu, P., Li, J., et al. (2003). Convergent evolution of perenniality in rice and sorghum. Proc. Natl. Acad. Sci. U.S.A. 100, 4050-4054. doi: 10.1073/pnas.0630531100

\section{FUNDING}

This work was funded by Yunnan Postdoctoral Sustentation Fund, China (2020 for XL) and National Natural Science Foundation of China (3U1302264 for FH).

\section{ACKNOWLEDGMENTS}

We would like to thank Lin Shao for his contribution in revising the language of our manuscript.

\section{SUPPLEMENTARY MATERIAL}

The Supplementary Material for this article can be found online at: https://www.frontiersin.org/articles/10.3389/fpls.2021. 576340/full\#supplementary-material

Iwano, M., Ito, K., Fujii, S., Kakita, M., Asano-Shimosato, H., Igarashi, M., et al. (2015). Calcium signalling mediates self-incompatibility response in the Brassicaceae. Nat. Plants 1:15128. doi: 10.1038/nplants.2015.128

Khush, G. S., Bacalangco, E., and Ogawa, T. (1990). A new gene for resistance to bacterial blight from O.longistaminata. Rice Genet. Newslett. 7, 121-122.

Leach, C. R. (1988). Detection and estimation of linkage for a co-dominant structural gene locus linked to a gametophytic self-incompatibility locus. Theor. Appl. Genet. 75, 882-888. doi: 10.1007/bf00258049

Li, J., Zhang, Y., Song, Y., Zhang, H., Fan, J., Li, Q., et al. (2017). Electrostatic potentials of the $S$-locus F-box proteins contribute to the pollen S specificity in self-incompatibility in Petunia hybrida. Plant J. 89, 45-57. doi: 10.1111/TPJ. 13318

Liang, M., Cao, Z., Zhu, A., Liu, Y., Tao, M., Yang, H., et al. (2020). Evolution of self-compatibility by a mutant Sm-RNase in citrus. Nat. Plants 6, 131-142. doi: 10.1038/s41477-020-0597-3

Liu, W., Fan, J., Li, J., Song, Y., Li, Q., Zhang, Y. E., et al. (2014). SCF ${ }^{S L F}$-mediated cytosolic degradation of S-RNase is required for cross-pollen compatibility in S-RNase-based self-Incompatibility in Petunia hybrida. Front. Genet. 5:228. doi: 10.3389/FGENE.2014.00228

Livak, K. J., and Schmittgen, T. D. (2001). Analysis of relative gene expression data using real-time quantitative PCR and the $2^{-\Delta \Delta C T}$ method. Methods 25, 402-408. doi: 10.1006/meth.2001.1262

Lundqvist, A. (1954). Studies on self-sterility in rye, Secale cereale L. Hereditas 40, 278-294. doi: 10.1111/J.1601-5223.1954.TB02973.X

Lundqvist, A. (1956). Self-incompatibility in rye. I. Genetic control in the diploid. Hereditas 42, 293-348. doi: 10.1111/j.1601-5223.1956.tb03021.x

Manzanares, C., Barth, S., Thorogood, D., Byrne, S. L., Yates, S., Czaban, A., et al. (2016). A gene encoding a DUF247 domain protein cosegregates with the $S$ self-incompatibility locus in perennial ryegrass. Mol. Biol. Evol. 33, 870-884. doi: 10.1093/MOLBEV/MSV335

McCormick, S. (1998). Self-incompatibility and other pollen-pistil interactions. Curr. Opin. Plant Biol. 1, 18-25. doi: 10.1016/S1369-5266(98)80122-2

McCubbin, A. G., and Kao, T. (2000). Molecular recognition and response in pollen and pistil interactions. Annu. Rev. Cell Dev. Biol. 16, 333-364. doi: 10.1146/ annurev.cellbio.16.1.333

Morishima, O. H. (1967). Variation in the breeding system of a wild rice, Oryza perennis. Evolution 21, 249-258. doi: 10.2307/2406673

Nayar, N. M. (1967). Prevalence of self-incompatibility in Oryza barthii Cheval.: Its bearing on the evolution of rice and related taxa. Genetica 38, 521-527. doi: 10.1007/BF01507481

Pandey, K. K. (1960). Evolution of gametophytic and sporophytic systems of selfincompatibility in angiosperms. Evolution 14, 98-115. doi: 10.2307/2405926 
Shinozuka, H., Cogan, N. O. I., Smith, K. F., Spangenberg, G. C., and Forster, J. W. (2010). Fine-scale comparative genetic and physical mapping supports mapbased cloning strategies for the self-incompatibility loci of perennial ryegrass (Lolium perenne L.). Plant Mol. Biol. 72, 343-355. doi: 10.1007/s11103-0099574-y

Silva, N. F., and Goring, D. R. (2001). Mechanisms of self-incompatibility in flowering plants. Cell. Mol. Life Sci. 58, 1988-2007. doi: 10.1007/PL00000832

Song, W., Wang, G., Chen, L., Kim, H. S., Pi, L., Holsten, T., et al. (1995). A receptor kinase-like protein encoded by the rice disease resistance gene, Xa21. Science 270, 1804-1806. doi: 10.1126/science.270.5243.1804

Takayama, S., and Isogai, A. (2005). Self-incompatibility in plants. Annu. Rev. Plant Biol. 56, 467-489. doi: 10.1146/annurev.arplant.56.032604.144249

Thorogood, D., Kaiser, W. J., Jones, J. G., and Armstead, I. (2002). Selfincompatibility in ryegrass 12 . Genotyping and mapping the $S$ and $Z$ loci of Lolium perenne L. Heredity 88, 385-390. doi: 10.1038/SJ.HDY.6800071

Thorogood, D., Yates, S., Manzanares, C., Skot, L., Hegarty, M., Blackmore, T., et al. (2017). A novel multivariate approach to phenotyping and association mapping of multi-locus gametophytic self-incompatibility reveals $S, Z$, and other loci in a perennial ryegrass (Poaceae) population. Front. Plant Sci. 8:1331. doi: 10.3389/fpls.2017.01331

Vaughan, D. A. (1994). The Wild Relatives of Rice; A Genetic Resources Handbook. Manila: International Rice Research Institute.

Vekemans, X., and Slatkin, M. (1994). Gene and allelic genealogies at a gametophytic self-incompatibility locus. Genetics 137, 1157-1165. doi: 10.1016/ S0959-8049(12)70009-6
Yang, B., Thorogood, D., Armstead, I. P., Franklin, F. C. H., and Barth, S. (2009). Identification of genes expressed during the self-incompatibility response in perennial ryegrass (Lolium perenne L.). Plant Mol. Biol. 70, 709-723. doi: 10. 1007/s11103-009-9501-2

Ye, M., Peng, Z., Tang, D., Yang, Z., Li, D., Xu, Y., et al. (2018). Generation of self-compatible diploid potato by knockout of S-RNase. Nat. Plants 4, 651-654. doi: 10.1038/s41477-018-0218-6

Zhang, D., and Zoe, A. (2009). Stamen specification and anther development in rice. Chin. Sci. Bull. 54, 2342-2353. doi: 10.1007/s11434-0090348-3

Zhang, Y., Zhang, S., Liu, H., Fu, B., Li, L., Xie, M., et al. (2015). Genome and comparative transcriptomics of African wild rice Oryza longistaminata provide insights into molecular mechanism of rhizomatousness and selfincompatibility. Mol. Plant 8, 1683-1686. doi: 10.1016/j.molp.2015.08.006

Conflict of Interest: The authors declare that the research was conducted in the absence of any commercial or financial relationships that could be construed as a potential conflict of interest.

Copyright $\odot 2021$ Lian, Zhang, Huang, Huang, Zhang and Hu. This is an open-access article distributed under the terms of the Creative Commons Attribution License (CC BY). The use, distribution or reproduction in other forums is permitted, provided the original author(s) and the copyright owner(s) are credited and that the original publication in this journal is cited, in accordance with accepted academic practice. No use, distribution or reproduction is permitted which does not comply with these terms. 